\title{
Aplicación de Bloomberg en el proceso de aprendizaje de los cursos de Contabilidad, Finanzas e Inversiones
}

\section{Aldo Augusto Martín Quintana Meza}

\author{
Departamento Académico de Ciencias Contables \\ Pontificia Universidad Católica del Perú (PUCP)
}

Desde la década de 1990, se dispone en Perú de sistemas de información económica y financiera con tecnología digital. Estos sistemas son usados de manera permanente por los profesionales de contabilidad, economía, finanzas, inversiones y otras carreras afines. Estas herramientas son Reuters y Bloomberg; esta última ha logrado un importante desarrollo tecnológico y alcance internacional. Asimismo, las principales empresas e instituciones del Perú, tanto públicas como privadas, hacen uso de estos sistemas de información para los procesos de análisis, investigación, planeamiento y toma decisiones. En este sentido, la aplicación de Bloomberg en el proceso de enseñanza y aprendizaje de los contenidos específicos de los cursos de Contabilidad, Finanzas e Inversiones se ha utilizado como innovación académica en el programa de pregrado en contabilidad de la Pontificia Universidad Católica del Perú. Por lo tanto, el propósito de la investigación es valorar la innovación realizada para conocer las opiniones y recopilar las experiencias de los estudiantes sobre el uso de Bloomberg, así como las recomendaciones como resultado de la innovación realizada.

Palabras clave: educación, docencia, contabilidad, finanzas, Bloomberg

\section{Applying Bloomberg in the Learning Process of Accounting, Finance and Investments Courses}

Since the 90's, economic and financial information systems with digital technology are available in Peru. These systems are permanently used by professionals in accounting, economics, finance, investments, and other related specialties. These tools are Reuters and Bloomberg, the latter being the one that has achieved significant technological development and international reach. Likewise, the main companies and institutions of Peru, both public and private, make use of these information systems for the processes of analysis, research, planning and decision making. In this sense, the application of Bloomberg in the teaching and learning process of the specific contents to the accounting, finance and investments courses has been used as academic innovation in the Accounting undergraduate program of Pontifical Catholic University From Peru. Therefore, the purpose of the research is to value the innovation made in order to know the opinions and collect the experiences of the students on the use of Bloomberg, and, at the same time, the recommendations as a result of the innovation made.

Keywords: education, teaching, accounting, finance, Bloomberg

\section{Aplicação da Bloomberg no processo de aprendizagem de cursos de contabilidade, finanças e investimentos}

Desde os anos 90, sistemas de informaçôes econômicas e financeiras com tecnologia digital estão disponíveis no Peru. Esses sistemas são utilizados permanentemente por profissionais de contabilidade, economia, finanças, investimentos 
e outras especialidades relacionadas. Essas ferramentas são a Reuters e a Bloomberg, sendo a última a que alcançou significativo desenvolvimento tecnológico e alcance internacional. Da mesma forma, as principais empresas e instituiçóes do Peru, públicas e privadas, utilizam esses sistemas de informação para os processos de análise, pesquisa, planejamento e tomada de decisão. Nesse sentido, a aplicação da Bloomberg no processo de ensino e aprendizagem de conteúdos específicos para os cursos de Contabilidade, Finanças e Investimentos tem sido utilizada como inovação acadêmica no curso de graduação em contabilidade da Pontifícia Universidade Católica do Peru. Portanto, o objetivo da pesquisa é valorizar a inovação realizada para conhecer as opinióes e coletar as experiências dos alunos sobre o uso da Bloomberg e, ao mesmo tempo, as recomendaçôes resultantes da inovaçáo realizada.

Palavras-chave: educação, ensino, contabilidade, finanças, Bloomberg

\section{Introducción y problema a abordar}

El proceso de enseñanza y aprendizaje requiere de una dinámica que permita interactuar de manera continua y actualizada entre la teoría y la realidad; de esta forma, se pueden contrastar las herramientas y contenidos de los cursos, y los modelos teóricos con la evidencia empírica. Desde la década de 1990, se dispone en el Perú de sistemas de información económica y financiera con tecnología digital. Estos sistemas son usados de manera permanente por los profesionales de contabilidad, economía, finanzas, inversiones y otras carreras afines. Estas herramientas son Reuters y Bloomberg. En particular, la última es la que ha logrado un mayor avance tecnológico, y difusión a nivel local y regional. De hecho, las principales empresas e instituciones tanto públicas como privadas de Perú hacen uso de estos sistemas de información para los procesos de análisis, investigación, planeamiento y toma de decisiones.

La importancia fundamental de la herramienta Bloomberg radica en el acercamiento a información actualizada, y bases de datos de empresas nacionales e internacionales. Por ello, ha sido usada como estrategia de innovación académica en los cursos de la carrera de contabilidad de la Pontificia Universidad Católica del Perú (PUCP) (Quintana, 2018). Los cursos seleccionados han sido (i) Estadística y Cálculo Financiero
(CON273) y (ii) Decisiones de Inversión (CON279) entre los semestres académicos del 2016-1 al 2019-1. En este artículo, se discuten los resultados del año 2016 correspondientes al primer año de implementación de la innovación académica.

En el proceso de enseñanza y aprendizaje de los contenidos específicos de los cursos de contabilidad, finanzas e inversiones, se ha utilizado la aplicación de Bloomberg como innovación académica en el programa de pregrado en contabilidad de la PUCP. Por lo tanto, el propósito de la investigación es valorar la innovación realizada para conocer y recopilar las opiniones y experiencias de los estudiantes sobre el uso de Bloomberg, así como posibles recomendaciones tras el resultado de la innovación realizada.

\section{Objetivos}

El objetivo de la investigación es evaluar el conocimiento y utilización de Bloomberg por parte de los estudiantes de las siguientes maneras:

- Como herramienta de análisis e investigación

- Para la toma de decisiones en las áreas de contabilidad, finanzas e inversiones

- Para el desarrollo de la habilidad de toma de decisiones

Contabilidad y Negocios (15) 29, 2020 / ISSN 1992-1896 


\section{Marco teórico}

Actualmente, el uso de la tecnología es importante en la instrucción de las finanzas (Duggal, 2006). Con el fin de promover una enseñanza activa, se sugiere el uso de laboratorios. Si bien los medios tradicionales de enseñanza de finanzas (como lecturas, portafolios o casos) son generalmente efectivos, podrían acarrear soluciones mecanizadas que no necesariamente garantizan el entendimiento de los conceptos trabajados por parte del estudiante (Duggal, 2006). Frente a ello, el uso de laboratorios es un poderoso recurso para mejorar las experiencias de aprendizaje de los estudiantes. Además, les provee de una importante herramienta de empleabilidad (Sharma, 2015), porque los acerca a experiencias reales. El uso de paquetes estadísticos y financieros se ha incrementado en la enseñanza de las finanzas internacionales (Sharma, 2015), así como el uso y análisis de información contable, económica y financiera en tiempo real.

Bloomberg (BPS, por sus siglas en inglés) es una herramienta que permite obtener información práctica del mercado financiero, ya que brinda datos cruzados en tiempo real, y realiza análisis de serie de tiempo y simulaciones de inversiones, entre otras razones (Bloomberg Finance L.P., 2020). Asimismo, esta herramienta ayuda al estudiante a desarrollar la toma de decisiones, pues permite analizar, cuestionar y evaluar una situación financiera tomando como base información de empresas reales (Sharma, 2015). Bloomberg es una herramienta creada alrededor de la década de 1980. Por su extendido uso en el mundo de las finanzas, ha llamado la atención del campo académico (Lei \& Li, 2012). Si bien Bloomberg solo ofrecía originalmente el servicio a la comunidad de inversiones, actualmente, ha expandido este servicio a instituciones académicas (Coe, 2007), por lo que es usado en diversas universidades a nivel mundial
(Scott, 2010). En el semestre 2015-1, la PUCP puso a disposición de los estudiantes esta plataforma, que permite el análisis y consulta de información financiera (Sistema de Bibliotecas, 2020). Esta herramienta puede proveer de un desarrollo de habilidades técnicas (Sharma, 2015). Se recomienda que el uso de este terminal esté alineado a objetivos y resultados de aprendizajes claros (Sharma, 2015).

\section{Metodología}

\section{Participantes}

En el año académico 2016, se encuestó a un total de 113 estudiantes, de los cuales 53 se encontraban en el curso Estadística y Cálculo Financiero; y 60, en el curso Decisiones de Inversión. Cabe señalar que el primer curso es requisito para el segundo, y ambos forman parte de la competencia específica del área de finanzas, que "Evalúa las decisiones de inversión y de financiamiento con la finalidad de contribuir a maximizar el valor de la organización" (Facultad de Ciencias Contables, 2020). Para el presente artículo, solo se presentan los resultados correspondientes al año académico 2016, que fue el primer año de implementación de la innovación.

\section{Instrumento}

Para conocer la aceptación del uso de Bloomberg en los estudiantes de Contabilidad, se usó una encuesta que constó de tres partes: 1) Uso del terminal antes del curso y reservas; 2) Ítems de satisfacción de Bloomberg; y 3) Observaciones y recomendaciones. En el apéndice 3, aparece el modelo de encuesta que se aplicó al final de cada semestre académico.

La primera parte contiene cinco preguntas: 1) si los alumnos habían usado Bloomberg antes de matricularse; 2) el lugar de uso de la aplicación; 3) el curso de 
uso de la aplicación; 4) el número de reservas realizadas durante el curso; y 5) el uso de dichas reservas (individual o grupal). La segunda parte constó de catorce ítems, que calificaban la importancia de Bloomberg del 1 al 10: el rango iba desde "muy en desacuerdo" (1) hasta "totalmente de acuerdo" (10). Se calificó la importancia otorgada al programa, así como las ventajas e influencia en el aprendizaje. Además, se presentaron afirmaciones sobre los servicios brindados por la PUCP y el rol del docente en el uso de Bloomberg. Finalmente, se les dio a los alumnos la libertad para que brinden su opinión sobre su experiencia con Bloomberg y recomendaciones si lo deseaban. Ambos aspectos fueron evaluados mediante dos preguntas abiertas.

\section{Resultados}

- Uso y reservas del terminal Bloomberg

En relación con la primera parte, se les preguntó a los participantes por el uso del terminal antes de matricularse en el curso, la cantidad de reservas del terminal durante el curso y el uso que le dieron a las reservas (individual o grupal). Del total de estudiantes encuestados, el 55\% de ellos reportó haber usado Bloomberg antes de la matrícula. Al analizar este ítem por cursos, se puede apreciar que la mayoría de estudiantes de Estadística y Cálculo Financiero (96\%) no había usado la plataforma con anterioridad. Por el contrario, la mayoría de estudiantes de Decisiones de Inversión (80\%) reportó haber usado la aplicación durante el ciclo anterior en el curso Estadística y Cálculo Financiero (ver figura 1).

Como se mencionó con anterioridad, el curso de Estadística y Cálculo Financiero es requisito para matricularse en Decisiones de Inversión. Esto podría explicar la diferencia entre los estudiantes de uno y otro curso. Además, se preguntó en qué curso(s) se había usado Bloomberg en caso de haberlo hecho. Como se muestra en la tabla 1, la mayoría de estudiantes que reportó haber usado la aplicación con anterioridad lo hizo en el curso Estadística y Cálculo Financiero; solo uno de los entrevistados usó Bloomberg fuera de la PUCP.

Figura 1. Porcentaje de estudiantes que utilizó el Bloomberg antes de matricularse en el curso

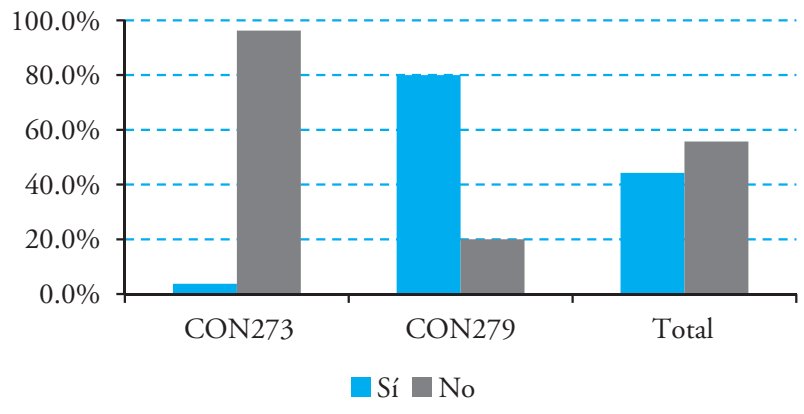

Tabla 1. Número y porcentaje de estudiantes que reportaron usar Bloomberg antes de matricularse

\begin{tabular}{|l|c|c|}
\hline \multicolumn{1}{|c|}{ Curso } & n & $\%$ \\
\hline Decisiones de Inversión & 3 & $6,0 \%$ \\
Estadística & 2 & $4,0 \%$ \\
Estadística y Cálculo Financiero & 44 & $88,0 \%$ \\
Fuera de la PUCP & 1 & $2,0 \%$ \\
\hline
\end{tabular}

La cifra del curso Decisiones de Inversión corresponde a estudiantes que están llevando el curso por segunda vez. Las cifras del curso Estadística corresponden al curso de Estudios Generales Letras (EEGGLL), previo al ingreso a la Facultad de Ciencias Contables.

Con relación a la cantidad de reservas, estas fueron variadas. Si bien las reservas oscilan entre 1 y 20, aproximadamente el $76 \%$ de estudiantes realizó entre 5 a 10 reservas durante el ciclo. Asimismo, cuatro estudiantes no realizaron reservas del terminal durante el ciclo (ver tabla 2). 
Tabla 2. Frecuencia de reservas de Bloomberg durante el ciclo

\begin{tabular}{|c|c|c|}
\hline Cantidad de uso & N & $\%$ \\
\hline 0 & 4 & $3,5 \%$ \\
1 & 1 & $0,9 \%$ \\
3 & 5 & $4,4 \%$ \\
4 & 9 & $8,0 \%$ \\
5 & 24 & $21,2 \%$ \\
6 & 19 & $16,8 \%$ \\
7 & 9 & $8,0 \%$ \\
8 & 13 & $11,5 \%$ \\
9 & 6 & $5,3 \%$ \\
10 & 15 & $13,3 \%$ \\
12 & 2 & $1,8 \%$ \\
15 & 1 & $0,9 \%$ \\
18 & 1 & $0,9 \%$ \\
20 & 4 & $3,5 \%$ \\
\hline
\end{tabular}

En relación con la forma de uso, de los estudiantes que reportaron haber reservado el terminal por lo menos una vez, $23 \%$ reportó no haberlo usado de manera individual. La frecuencia de uso individual oscila entre 1 a 17 , y se concentra mayoritariamente entre 1 y 4 reservas para uso individual (ver tabla 3 ).

Tabla 3. Frecuencia de reservas para uso individual

\begin{tabular}{|c|c|c|}
\hline & $\mathbf{n}$ & $\%$ \\
\hline 0 & 22 & $23,0 \%$ \\
1 & 25 & $22,1 \%$ \\
2 & 22 & $19,5 \%$ \\
3 & 12 & $10,6 \%$ \\
4 & 12 & $10,6 \%$ \\
5 & 5 & $4,4 \%$ \\
6 & 2 & $1,8 \%$ \\
7 & 2 & $1,8 \%$ \\
8 & 1 & $0,9 \%$ \\
10 & 1 & $0,9 \%$ \\
12 & 1 & $0,9 \%$ \\
13 & 1 & $0,9 \%$ \\
15 & 2 & $1,8 \%$ \\
17 & 1 & $0,9 \%$ \\
\hline Total & 109 & $100,0 \%$ \\
\hline
\end{tabular}

En relación con las reservas para uso grupal, la distribución varía entre 1 a 10 reservaciones. La mayoría de estudiantes (74\%) reservó el terminal entre 3 y 6 veces para uso grupal (ver tabla 4). Sobre la base de la información de las tablas 2, 3 y 4, se observa que aproximadamente el $96 \%$ de los estudiantes ha usado Bloomberg por lo menos una vez durante el ciclo y, en su mayoría, de forma grupal.

Tabla 4. Frecuencia de reservas para uso grupal

\begin{tabular}{|c|c|c|}
\hline & $\mathbf{n}$ & $\%$ \\
\hline 1 & 2 & $1,8 \%$ \\
2 & 9 & $8,3 \%$ \\
3 & 21 & $19,3 \%$ \\
4 & 21 & $19,3 \%$ \\
5 & 27 & $24,8 \%$ \\
6 & 12 & $11,0 \%$ \\
7 & 3 & $2,8 \%$ \\
8 & 10 & $9,2 \%$ \\
9 & 3 & $2,8 \%$ \\
10 & 1 & $0,9 \%$ \\
\hline Total & 109 & $100,0 \%$ \\
\hline
\end{tabular}

A. Opiniones sobre el uso

A los estudiantes se les consultó sobre la importancia otorgada al programa, así como sobre sus ventajas e influencia en su aprendizaje. Además, se les presentaron afirmaciones sobre los servicios brindados por la PUCP y el rol del docente en el uso de Bloomberg. Para ello, se les presentaron 14 afirmaciones, que debían ser valoradas del 1 al 10: 1 equivalía a "muy en desacuerdo"; y 10, a "totalmente de acuerdo". Para el reporte de resultados de esta sección, los ítems se agruparon de la siguiente manera:

- Importancia del BPS: ítems 1, 2 y 7

- Ventajas del BPS: ítems 3y 4

- Consideraciones de uso: 5 y 8

- Importancia del BPS para el aprendizaje: 6, 9 y 10

- Servicio brindado por la PUCP: 11 y 12 
- El rol del docente en el uso de BPS: 13 y 14

En relación con la importancia de Bloomberg, la mayoría de estudiantes reportó estar de acuerdo con las afirmaciones vinculadas a la importancia de conocer esta herramienta y su uso para la toma de decisiones (ver tabla 5).

Con relación a las ventajas del Bloomberg, la mayoría de estudiantes está de acuerdo en señalar que esta herramienta brinda datos nacionales e internacionales, y permite contrastar la teoría con la práctica (ver tabla 6). Asimismo, la mayoría de estudiantes señaló estar de acuerdo con las afirmaciones relacionadas a la importancia de la certificación oficial del Bloomberg y la elaboración de materiales en español (ver tabla 7). Con respecto a las afirmaciones relacionadas al uso de Bloomberg como apoyo para la mejora de los aprendizajes del curso, la mayoría de estudiantes reportó encontrase totalmente de acuerdo en señalar que sí los ha ayudado a aprender mejor y que es fundamental para su formación profesional (ver tabla 8).

Los puntajes otorgados al servicio que brinda la PUCP es menor en relación con los demás ítems, en particular a la disposición de terminales en el campus. Sin embargo, los estudiantes reportaron una mejor valoración a la atención que reciben (ver tabla 9).

Tabla 5. Estadísticos descriptivos de los ítems relacionados a la importancia del BPS

\begin{tabular}{|c|c|c|c|c|c|}
\hline & Media & Mediana & Desviación estándar & Mínimo & Máximo \\
\hline $\begin{array}{l}\text { Es importante que los alumnos de la carrera de contabilidad } \\
\text { conozcan y utilicen el Bloomberg como herramienta de análisis } \\
\text { e investigación. }\end{array}$ & 9,59 & 10,00 & 1,107 & 1 & 10 \\
\hline $\begin{array}{l}\text { Es importante que los alumnos de la carrera de contabilidad } \\
\text { conozcan y utilicen el Bloomberg para la toma de decisiones. }\end{array}$ & 9,54 & 10,00 & 0,916 & 6 & 10 \\
\hline $\begin{array}{l}\text { Es importante que los alumnos de la carrera de contabilidad } \\
\text { conozcan el Bloomberg University. }\end{array}$ & 9,41 & 10,00 & 1,227 & 4 & 10 \\
\hline
\end{tabular}

Tabla 6. Estadísticos descriptivos de los ítems relacionados a las ventajas del BPS

\begin{tabular}{|c|c|c|c|c|c|}
\hline & Media & Mediana & Desviación estándar & Mínimo & Máximo \\
\hline $\begin{array}{l}\text { Una de las principales ventajas de contar con esta herramienta es } \\
\text { que se dispone con bases de datos de empresas, y datos naciona- } \\
\text { les e internacionales. }\end{array}$ & 9,27 & 10,00 & 1,245 & 4 & 10 \\
\hline $\begin{array}{l}\text { El uso de Bloomberg en las clases y evaluaciones permite con- } \\
\text { trastar la teoría con la evidencia empírica con un enfoque tanto } \\
\text { doméstico como internacional. }\end{array}$ & 9,52 & 10,00 & ,927 & 6 & 10 \\
\hline
\end{tabular}

Tabla 7. Estadísticos descriptivos de los ítems relacionados a consideraciones del BPS

\begin{tabular}{|c|c|c|c|c|c|}
\hline & Media & Mediana & Desviación estándar & Mínimo & Máximo \\
\hline $\begin{array}{l}\text { Se debería elaborar una librería de videos en español sobre el uso } \\
\text { de Bloomberg y sus aplicaciones en la carrera de contabilidad. }\end{array}$ & 9,61 & 10,00 & 990 & 4 & 10 \\
\hline $\begin{array}{l}\text { Es importante que los alumnos realicen la certificación oficial del } \\
\text { Bloomberg. }\end{array}$ & 8,91 & 10,00 & 1,704 & 3 & 10 \\
\hline
\end{tabular}


Tabla 8. Estadísticos descriptivos de los ítems relacionados a la contribución del BPS al aprendizaje

\begin{tabular}{|c|c|c|c|c|c|}
\hline & Media & Mediana & Desviación estándar & Mínimo & Máximo \\
\hline $\begin{array}{l}\text { El uso de la información proporcionada por el Bloomberg en } \\
\text { las sesiones de clase contribuyeron al mejor aprendizaje de los } \\
\text { contenidos del sílabo. }\end{array}$ & 8,95 & 10,00 & 1,552 & 2 & 10 \\
\hline $\begin{array}{l}\text { Creo que, si no hubiera usado el Bloomberg durante el ciclo, } \\
\text { habría aprendido menos. }\end{array}$ & 8,57 & 9,00 & 1,728 & 3 & 10 \\
\hline $\begin{array}{l}\text { Considero que lo aprendido en este curso con el uso de Bloom- } \\
\text { berg es fundamental para mi formación profesional. }\end{array}$ & 9,07 & 10,00 & 1,425 & 3 & 10 \\
\hline
\end{tabular}

Tabla 9. Estadísticos descriptivos de los ítems relacionados al servicio brindado por la PUCP

\begin{tabular}{|c|c|c|c|c|c|}
\hline & Media & Mediana & Desviación estándar & Mínimo & Máximo \\
\hline $\begin{array}{l}\text { La disposición y el número de terminales de Bloomberg en el } \\
\text { campus San Miguel es adecuada. }\end{array}$ & 6,20 & 7,00 & 2,690 & 1 & 10 \\
\hline La atención de los terminales de Bloomberg es adecuada. & 7,56 & 8,00 & 2,134 & 2 & 10 \\
\hline
\end{tabular}

Tabla 10. Estadísticos descriptivos de los ítems relacionados al rol del docente en el uso de BPS

\begin{tabular}{|c|c|c|c|c|c|}
\hline & Media & Mediana & Desviación estándar & Mínimo & Máximo \\
\hline $\begin{array}{l}\text { El docente del curso debería usar más el terminal de Bloomberg } \\
\text { durante las clases. }\end{array}$ & 8,09 & 8,00 & 1,806 & 3 & 10 \\
\hline $\begin{array}{l}\text { El docente del curso debería realizar al menos un taller durante } \\
\text { el ciclo sobre el uso de Bloomberg. }\end{array}$ & 9,27 & 10,00 & 1,722 & 2 & 10 \\
\hline
\end{tabular}

Finalmente, los estudiantes están altamente de acuerdo con que los docentes utilicen Bloomberg durante las clases y en la elaboración de talleres fuera de esta (ver tabla 10).

En general, los puntajes otorgados por los estudiantes han sido mayores a 8, con excepción de los ítems relacionados al servicio brindado por la PUCP (ver apéndice 1 y apéndice 3 ).

\section{B. Comentarios}

Con relación a los comentarios sobre la experiencia en el uso de Bloomberg, la mayoría de estudiantes manifestó que el uso del terminal les parece sencillo o normal. Sin embargo, en lo comentarios, señalaron haber tenido dificultades al inicio, aunque pudieron solucionarlas con la práctica o con la ayuda de otros compañeros ${ }^{1}$.

\footnotetext{
1 "Al principio fue difícil; luego, con la frecuencia de ir con mi grupo, pude aprender y, por lo que pude ver, no era muy difícil" (comentario de estudiante de Estadística y Cálculo Financiero). "Ha sido un poco complicado al inicio, pero, con la práctica, todo se vuele más sencillo" (comentario de estudiante de Decisiones de Inversión).
} 
Entre las limitaciones o problemas del terminal, los estudiantes señalaron tener inconvenientes en los códigos, como con la terminología ${ }^{2}$; y las abreviaturas, pues la aplicación está en inglés ${ }^{3}$. Sin embargo, después de que los estudiantes empiezan a usar más el sistema, resuelven estos inconvenientes con la práctica $^{4}$. Otra de las limitaciones señaladas es que la PUCP no cuenta con un laboratorio ni con computadoras suficientes para los estudiantes, por lo que los alumnos tienen que hacer reservaciones y la práctica se hace difícil ${ }^{5}$. Además, los encuestados señalaron que no hay una guía con pasos básicos o tutoriales que les ayude a comenzar a usar el terminal ${ }^{6}$.

Como sugerencia más recurrente de parte de los estudiantes, aparece la propuesta de realizar talleres en los que se les brinde conceptos básicos o señalen los principales comandos ${ }^{7}$. En relación con el curso o etapa en la que se debe comenzar a usar el terminal, las opiniones son variadas. Por un lado, algunos estudiantes señalan que deberían brindarse talleres y dar a conocer el terminal desde Estudios Generales Letras por medio de cursos electivos y talleres dirigidos a estudiantes afines a las carreras de negocio o gestión ${ }^{8}$. Además, se sugiere que estos cursos se realicen a modo de introducción para que luego los estudiantes lleguen preparados a la Facultad de Ciencias Contables?. Por otro lado, un grupo de estudiantes considera que el manejo de Bloomberg debería enseñarse recién en cursos de facultad, debido a que no es de interés de todos los estudiantes de Estudios Generales Letras ${ }^{10}$. Además, sostienen que, de haber mayor demanda del terminal, habrá mayor dificultad para la reserva de computadoras que cuentan con él ${ }^{11}$.

\section{Conclusiones}

- Los estudiantes encuestados, en su mayoría, han usado Bloomberg durante el ciclo. Además, no se reportó el uso de Bloomberg fuera de los cursos analizados.

2 "Al ser un tema nuevo con términos distintos puede confundir al usuario" (comentario de estudiante de Decisiones de Inversión).

3 "Es complicado aprender a usarlo, ya que utiliza abreviaturas en inglés, que son desconocidas cuando se usa por primera vez" (comentario de estudiante de Estadística y Cálculo Financiero). "Es difícil, ya que las funciones están en inglés y no toda la información esta traducida" (comentario de estudiante de Decisiones de Inversión).

4 "Es normal, ya que requiere practica y curiosidad para poder llegar a dominarlo" (comentario de estudiante de Estadística y Cálculo Financiero).

5 "Hay muy pocos terminales en la PUCP, están muy copados y se han reducido a solo un monitor, lo que dificulta la reservación" (comentario de estudiante de Decisiones de Inversión). “Con la práctica es más fácil su uso; por eso, debe haber más terminales” (comentario de estudiante de Estadística y Cálculo Financiero).

6 "No hay tutoriales sobre este manejo" (comentario de estudiante de Estadística y Cálculo Financiero).

7 "Considero pertinente hacer un taller para los alumnos que recién ingresan a facultad para explicarles su funcionamiento" (comentario de estudiante de Estadística y Cálculo Financiero). "Es difícil aprender las funciones; por tanto, debería de realizase más talleres para saber sus funciones con diccionario e interpretación” (comentario de estudiante de Decisiones de Inversión).

8 "En Estudios Generales Letras, podrían darse talleres opcionales para los que optan por carreras de Contabilidad" (comentario de estudiante de Estadística y Cálculo Financiero).

9 "Sería bueno tener talleres desde Estudios Generales Letras para que se vayan adecuado a los cursos de finanzas de la facultad, ya que la transición es dura" (comentario de estudiante de Decisiones e Inversión).

10 "Solo en cursos de facultad, ya que requiere conocimientos previos. Debería haber más talleres" (comentario de estudiante de Decisiones de Inversión).

11 “(...) si se extiende a Estudios Generales Letras, los terminales estarán llenos y no se usen [usarán] adecuadamente” (comentario de estudiante de Estadística y Cálculo Financiero). 
- Durante el ciclo, los estudiantes han debido reservar los terminales con más frecuencia. Solo cuatro de los estudiantes encuestados no los ha usado durante el ciclo.

- En general, los estudiantes consideran importante el uso del terminal como herramienta de análisis e investigación, y consideran que debe ponerse en conocimiento la existencia de Bloomberg. Algunos señalaron que no sabían de su existencia antes del curso $^{12}$. Aun así, la mayoría de estudiantes consideró importante esta herramienta para la toma de decisiones empresariales. Esto coincide con lo señalado por Sharma (2015), que menciona que Bloomberg provee al estudiante de un ambiente que le permite apreciar el mundo real de las finanzas y, por ende, facilita la toma de decisiones.

- La mayoría de estudiantes señaló que la principal ventaja de Bloomberg es que dispone de datos nacionales e internacionales, que permitirían contrastar la teoría con la práctica.

- Los estudiantes coincidieron en señalar que el uso de Bloomberg favorece su aprendizaje. Asimismo, consideraron que, de no haber realizado operaciones en el terminal, hubieran aprendido menos. Como señala Sharma (2015), el uso del terminal promueve un aprendizaje más activo y desarrolla el potencial de los estudiantes.

- Con relación a cómo se gestiona el terminal en la PUCP, la disposición de terminales es el aspecto menos valorado. Ello se puede deber al costo que requiere la adquisición de terminales. Sin embargo, a largo plazo (considerando costo y beneficio), la inversión en más terminales puede traer mayores beneficios al estudiante (Coe, 2007).

- La importancia de la preparación del docente y el uso de Bloomberg en clase también es una recomendación mencionada por los estudiantes ${ }^{13}$. Este punto es fundamental, pues, como señala Sharma (2015), la clave para un adecuado uso del terminal es tener un equipo de profesionales motivados y entrenados en el uso del terminal. Si bien es una herramienta útil, el sistema de navegación no es fácil, por lo que requiere de guía y entrenamiento (Scott, 2010). Dicho entrenamiento debe recaer en docentes y jefes de prácticas capacitados.

- Entre las dificultades más reportadas, están las abreviaturas y códigos. Lei y Li (2012) encontraron la misma dificultad, por lo que identificaron e ilustraron funciones claves para preparar a estudiantes en el análisis y reporte de datos. Una vez que se familiarizaron con las claves, procedieron a explorar más funciones en el terminal, mientras se realizó un análisis en paralelo. Un proceso similar podría potenciar el uso de la herramienta incluso fuera de la PUCP y facilitar la comprensión de términos reportado por los estudiantes.

- Es importante anotar que, luego de la implementación de la innovación realizada en el año 2016, el uso de la herramienta se ha ido extendiendo a otros cursos de finanzas dentro y fuera de la carrera de contabilidad de la PUCP, tanto en pregrado como en posgrado. En gran medida, eso se debe a la valoración positiva que le otorgan los estudiantes

\footnotetext{
12 “(...) no es muy conocido, no sabía que existía” (comentario de estudiante de Estadística y Cálculo Financiero).

13 "El jefe de prácticas, durante clase práctica, no usa Bloomberg. Debería haber más clase para el uso de este" (comentario de estudiante de Decisiones de Inversión). "Debería haber asistentes mejor capacitados" (comentario de estudiante de Estadística y Cálculo Financiero)
} 
a la aplicación de Bloomberg en el proceso de aprendizaje de los cursos de Contabilidad, Economía, Finanzas e Inversiones.

\section{Recomendaciones}

Entre las recomendaciones de los estudiantes, se sugería la elaboración de material audiovisual de soporte para el uso del aplicativo, que esté disponible en el aula virtual de los cursos. En respuesta a ello, se elaboró material audiovisual de soporte al estudiante, el cual está disponible en el siguiente enlace: https:// drive.google.com/drive/folders/0B9DfZC0VzfTfSnV ReVhTTnl1TUk?usp=sharing

\section{Referencias bibliográficas}

Bloomberg Finance L.P. (2020). Universidades. Servicio Bloomberg Professional. Recuperado de https://www. bloomberg.com/latam/expertos/universidades/

Coe, T. (2007). Using the Bloomberg Professional System for Finance Classes. Journal of Economics and Finance Education, (33), 48-62.

Duggal, R. (2006). The Costs and Benefits of a Finance Lab. Journal of College Teaching and Learning, 3(11), 81-83. https://doi.org/10.19030/tlc.v3i11.1662

Estudios Generales Letras (2020). El desarrollo de competencias genéricas y los perfiles de ingreso y egreso. Recuperado de http://facultad.pucp.edu.pe/generales-letras/perfildel-estudiante/presentacion/

Facultad de Ciencias Contables (2020). Perfil del egresado. Recuperado de http://facultad.pucp.edu.pe/cienciascontables/egresados/perfil-del-egresado/

Lei, A. y Li, H. (2012). Using Bloomberg Terminals in a Security Analysis and Portfolio Management Course. Journal of Economics and Finance Education, 11 (2), 17-33. https://doi.org/10.2139/ssrn.1999066

Quintana, A. (2018). Aplicación de Bloomberg en el proceso de aprendizaje de los cursos de contabilidad, finanzas e inversiones. Cuadernos de Innovación en la Docencia Universitaria, 63-71.

Scott, R. (2010). Bloomberg 101. Journal of Financial Education, (36), 80-88.

Sharma, C. (2015). Use of Bloomberg Professional in Support of Finance and Economics Teaching. Journal Cogent Economics and Finance, (3). https://doi.org/1 $0.1080 / 23322039.2015 .1115618$

Sistema de Bibliotecas (2020). Bloomberg Professional. Recuperado de http://biblioteca.pucp.edu.pe/recursoelectronico/bloomberg-professional/

Fecha de recepción: 05 de noviembre de 2019 Fecha de aceptación: 12 de febrero de 2020 Correspondencia: aaquintana@pucp.edu.pe 


\section{Apéndices}

\section{Apéndice 1. Distribución de respuestas por ítem}

\section{Apéndice 1A. Importancia del Bloomberg}

P1 Es importante que los alumnos de la carrera de Contabilidad conozcan y utilicen el Bloomberg como herramienta de análisis e investigación.

\begin{tabular}{|c|c|c|}
\hline Puntaje & $\mathbf{n}$ & $\%$ \\
\hline 1 & 1 & $0,9 \%$ \\
6 & 1 & $0,9 \%$ \\
7 & 1 & $0,9 \%$ \\
8 & 10 & $8,8 \%$ \\
9 & 10 & $8,8 \%$ \\
10 & 90 & $79,6 \%$ \\
Total & 113 & $100,0 \%$ \\
\hline
\end{tabular}

P2 Es importante que los alumnos de la carrera de Contabilidad conozcan y utilicen el Bloomberg para la toma de decisiones.

\begin{tabular}{|c|c|c|}
\hline Puntaje & $\mathbf{n}$ & $\%$ \\
\hline 6 & 2 & $1,8 \%$ \\
7 & 4 & $3,5 \%$ \\
8 & 9 & $8,0 \%$ \\
9 & 14 & $12,4 \%$ \\
10 & 84 & $74,3 \%$ \\
Total & 113 & $100,0 \%$ \\
\hline
\end{tabular}

P7 Es importante que los alumnos de la carrera de Contabilidad conozcan el Bloomberg University.

\begin{tabular}{|c|c|c|}
\hline Puntaje & $\mathbf{n}$ & $\%$ \\
\hline 4 & 2 & $1,8 \%$ \\
5 & 1 & $0,9 \%$ \\
6 & 3 & $2,7 \%$ \\
8 & 12 & $10,6 \%$ \\
9 & 13 & $11,5 \%$ \\
10 & 81 & $71,7 \%$ \\
Válidos & 112 & $99,1 \%$ \\
Perdidos & 1 & $0,9 \%$ \\
Total & 113 & $100,0 \%$ \\
\hline
\end{tabular}

\section{Apéndice 1B. Ventajas del Bloomberg}

P3 Una de las principales ventajas de contar con esta herramienta es que se dispone con base de datos de empresas, datos nacionales e internacionales.

\begin{tabular}{|c|c|c|}
\hline Puntaje & $\mathbf{n}$ & $\%$ \\
\hline 4 & 2 & $1,8 \%$ \\
5 & 1 & $0,9 \%$ \\
6 & 1 & $0,9 \%$ \\
7 & 3 & $2,7 \%$ \\
8 & 20 & $17,7 \%$ \\
9 & 12 & $10,6 \%$ \\
10 & 73 & $64,6 \%$ \\
Válidos & 112 & $99,1 \%$ \\
Perdidos & 1 & $0,9 \%$ \\
Total & 113 & $100,0 \%$ \\
\hline
\end{tabular}

P4 El uso de Bloomberg en las clases y evaluaciones permite contrastar la teoría con la evidencia empírica con un enfoque tanto doméstico como internacional.

\begin{tabular}{|c|c|c|}
\hline Puntaje & $\mathbf{n}$ & $\%$ \\
\hline 6 & 2 & $1,8 \%$ \\
7 & 3 & $2,7 \%$ \\
8 & 13 & $11,5 \%$ \\
9 & 11 & $9,7 \%$ \\
10 & 84 & $74,3 \%$ \\
Total & 113 & $100,0 \%$ \\
\hline
\end{tabular}

\section{Apéndice 1C. Consideraciones en el uso del Bloomberg}

P5 Se debería elaborar una librería de videos en español sobre el uso de Bloomberg y sus aplicaciones en la carrera de Contabilidad.

\begin{tabular}{|c|c|c|}
\hline Puntaje & $\mathbf{n}$ & $\%$ \\
\hline 4 & 1 & $0,9 \%$ \\
5 & 1 & $0,9 \%$ \\
7 & 3 & $2,7 \%$ \\
8 & 7 & $6,2 \%$ \\
9 & 10 & $8,8 \%$ \\
10 & 90 & $79,6 \%$ \\
Válidos & 112 & $99,1 \%$ \\
Perdidos & 1 & $0,9 \%$ \\
Total & 113 & $100,0 \%$ \\
\hline
\end{tabular}


P8 Es importante que los alumnos realicen la certificación oficial del Bloomberg.

\begin{tabular}{|c|c|c|}
\hline Puntaje & $\mathbf{n}$ & $\%$ \\
\hline 3 & 1 & $0,9 \%$ \\
4 & 3 & $2,7 \%$ \\
5 & 5 & $4,4 \%$ \\
6 & 4 & $3,5 \%$ \\
7 & 5 & $4,4 \%$ \\
8 & 13 & $11,5 \%$ \\
9 & 16 & $14,2 \%$ \\
10 & 66 & $58,4 \%$ \\
Total & 113 & $100,0 \%$ \\
\hline
\end{tabular}

\section{Apéndice 1D. Importancia del Bloomberg para el aprendizaje}

P6 El uso de la información proporcionada por el Bloomberg en las sesiones de clase contribuyeron al mejor aprendizaje de los contenidos del sílabo.

\begin{tabular}{|c|c|c|}
\hline Puntaje & $\mathbf{n}$ & $\%$ \\
\hline 2 & 1 & $0,9 \%$ \\
4 & 1 & $0,9 \%$ \\
5 & 2 & $1,8 \%$ \\
6 & 4 & $3,5 \%$ \\
7 & 12 & $10,6 \%$ \\
8 & 15 & $13,3 \%$ \\
9 & 13 & $11,5 \%$ \\
10 & 65 & $57,5 \%$ \\
Total & 113 & $100,0 \%$ \\
\hline
\end{tabular}

P9 Creo que, si no hubiera usado el Bloomberg durante el ciclo, habría aprendido menos.

\begin{tabular}{|c|c|c|}
\hline Puntaje & $\mathbf{n}$ & $\%$ \\
\hline 3 & 1 & $0,9 \%$ \\
4 & 3 & $2,7 \%$ \\
5 & 5 & $4,4 \%$ \\
6 & 3 & $2,7 \%$ \\
7 & 17 & $15,0 \%$ \\
8 & 15 & $13,3 \%$ \\
9 & 17 & $15,0 \%$ \\
10 & 51 & $45,1 \%$ \\
Validos & 112 & $99,1 \%$ \\
Perdidos & 1 & $0,9 \%$ \\
Total & 113 & $100,0 \%$ \\
\hline
\end{tabular}

P10 Considero que lo aprendido en este curso con el uso de Bloomberg es fundamental para mi formación profesional.

\begin{tabular}{|c|c|c|}
\hline Puntaje & n & $\%$ \\
\hline 3 & 1 & $0,9 \%$ \\
5 & 3 & $2,7 \%$ \\
6 & 3 & $2,7 \%$ \\
7 & 7 & $6,2 \%$ \\
8 & 20 & $17,7 \%$ \\
9 & 10 & $8,8 \%$ \\
10 & 69 & $61,1 \%$ \\
Total & 113 & $100,0 \%$ \\
\hline
\end{tabular}

\section{Apéndice 1E. Servicios brindados por la PUCP}

P11 La disposición y el número de terminales de Bloomberg en el campus San Miguel es adecuada.

\begin{tabular}{|c|c|c|}
\hline Puntaje & $\mathbf{n}$ & $\%$ \\
\hline 1 & 11 & $9,7 \%$ \\
2 & 3 & $2,7 \%$ \\
3 & 4 & $3,5 \%$ \\
4 & 11 & $9,7 \%$ \\
5 & 14 & $12,4 \%$ \\
6 & 10 & $8,8 \%$ \\
7 & 19 & $16,8 \%$ \\
8 & 19 & $16,8 \%$ \\
9 & 7 & $6,2 \%$ \\
10 & 15 & $13,3 \%$ \\
Total & 113 & $100,0 \%$ \\
\hline
\end{tabular}

P12 La atención de los terminales de Bloomberg es adecuada.

\begin{tabular}{|c|c|c|}
\hline Puntaje & $\mathbf{n}$ & $\%$ \\
\hline 2 & 3 & $2,7 \%$ \\
3 & 4 & $3,5 \%$ \\
4 & 5 & $4,4 \%$ \\
5 & 6 & $5,3 \%$ \\
6 & 11 & $9,7 \%$ \\
7 & 20 & $17,7 \%$ \\
8 & 20 & $17,7 \%$ \\
9 & 17 & $15,0 \%$ \\
10 & 26 & $23,0 \%$ \\
Válidos & 112 & $99,1 \%$ \\
Perdidos & 1 & $0,9 \%$ \\
Total & 113 & $100,0 \%$ \\
\hline
\end{tabular}




\section{Apéndice 1F. Rol del docente en el uso de Bloomberg}

P13 El docente del curso debería usar más el terminal de Bloomberg durante las clases.

\begin{tabular}{|c|c|c|}
\hline Puntaje & $\mathbf{n}$ & $\%$ \\
\hline 3 & 3 & $2,7 \%$ \\
5 & 10 & $8,8 \%$ \\
6 & 5 & $4,4 \%$ \\
7 & 21 & $18,6 \%$ \\
8 & 25 & $22,1 \%$ \\
9 & 12 & $10,6 \%$ \\
10 & 37 & $32,7 \%$ \\
Total & 113 & $100,0 \%$ \\
\hline
\end{tabular}

P14 El docente del curso debería realizar al menos un taller durante el ciclo sobre el uso de Bloomberg.

\begin{tabular}{|c|c|c|}
\hline Puntaje & $\mathbf{n}$ & $\%$ \\
\hline 2 & 3 & $2,7 \%$ \\
5 & 3 & $2,7 \%$ \\
6 & 4 & $3,5 \%$ \\
7 & 4 & $3,5 \%$ \\
8 & 5 & $4,4 \%$ \\
9 & 6 & $5,3 \%$ \\
10 & 88 & $77,9 \%$ \\
Total & 113 & $100,0 \%$ \\
\hline
\end{tabular}

\section{Apéndice 2. Estadísticos descriptivos según ítems}

\section{Apéndice 2A. Relacionados a la importancia del Bloomberg}

\begin{tabular}{|l|c|c|c|c|c|c|}
\hline \multicolumn{1}{|c|}{ Ítems } & n & Media & Mediana & Desviación estándar & Mínimo & Máximo \\
\hline $\begin{array}{l}\text { 1. Es importante que los alumnos de la carrera de Contabilidad } \\
\text { conozcan y utilicen el Bloomerg como herramienta de análisis e } \\
\text { investigación. }\end{array}$ & 113 & 9,59 & 10,00 & 1,107 & 1 & 10 \\
\hline $\begin{array}{l}\text { 2. Es importante que los alumnos de la carrera de Contabilidad } \\
\text { conozcan y utilicen el Bloomberg para la toma de decisiones. }\end{array}$ & 113 & 9,54 & 10,00 &, 916 & 6 & 10 \\
\hline $\begin{array}{l}\text { 7. Es importante que los alumnos de la carrera de contabilidad } \\
\text { conozcan el Bloomberg University. }\end{array}$ & 112 & 9,41 & 10,00 & 1,227 & 4 & 10 \\
\hline
\end{tabular}

\section{Apéndice 2B. Relacionados a las ventajas del Bloomberg}

\begin{tabular}{|l|c|c|c|c|c|c|}
\hline \multicolumn{1}{|c|}{ Ítems } & $\mathbf{n}$ & Media & Mediana & Desviación estándar & Mínimo & Máximo \\
\hline $\begin{array}{l}\text { 3. Una de las principales ventajas de contar con esta herramienta } \\
\text { es que se dispone con BD de empresas, datos nacionales e inter- } \\
\text { nacionales. }\end{array}$ & 112 & 9,27 & 10,00 & 1,245 & 4 & 10 \\
\hline $\begin{array}{l}\text { 4. El uso de Bloomberg en las clases y evaluaciones permite con- } \\
\text { trastar la teoría con la evidencia empírica con un enfoque tanto } \\
\text { doméstico como internacional. }\end{array}$ & 113 & 9,52 & 10,00 &, 927 & 6 & 10 \\
\hline
\end{tabular}

\section{Apéndice 2C. Relacionados a las condiciones de uso del Bloomberg}

\begin{tabular}{|l|c|c|c|c|c|c|}
\hline \multicolumn{1}{|c|}{ Ítems } & $\mathbf{n}$ & Media & Mediana & Desviación estándar & Mínimo & Máximo \\
\hline $\begin{array}{l}\text { 5. Se debería elaborar una librería de videos en español sobre el uso } \\
\text { de Bloomberg y sus aplicaciones en la carrera de Contabilidad. }\end{array}$ & 112 & 9,61 & 10,00 &, 990 & 4 & 10 \\
\hline $\begin{array}{l}\text { 8. Es importante que los alumnos realicen la certificación oficial } \\
\text { del Bloomberg. }\end{array}$ & 113 & 8,91 & 10,00 & 1,704 & 3 & 10 \\
\hline
\end{tabular}




\section{Apéndice 2E. Relacionados a la importancia del Bloomberg para su aprendizaje}

\begin{tabular}{|c|c|c|c|c|c|c|}
\hline Ítems & n & Media & Mediana & Desviación estándar & Mínimo & Máximo \\
\hline $\begin{array}{l}\text { 6. El uso de la información proporcionada por el Bloomberg en } \\
\text { las sesiones de clase contribuyeron al mejor aprendizaje de los } \\
\text { contenidos del sílabo. }\end{array}$ & 113 & 8,95 & 10,00 & 1,552 & 2 & 10 \\
\hline $\begin{array}{l}\text { 9. Creo que, si no hubiera usado el Bloomberg durante el ciclo, } \\
\text { habría aprendido menos. }\end{array}$ & 112 & 8,57 & 9,00 & 1,728 & 3 & 10 \\
\hline $\begin{array}{l}\text { 10. Considero que lo aprendido en este curso con el uso de } \\
\text { Bloomberg es fundamental para mi formación profesional. }\end{array}$ & 113 & 9,07 & 10,00 & 1,425 & 3 & 10 \\
\hline
\end{tabular}

\section{Apéndice 2F. Relacionados a los servicios brindados por la PUCP para el uso del Bloomberg}

\begin{tabular}{|l|c|c|c|c|c|c|}
\hline \multicolumn{1}{|c|}{ Ítems } & $\mathbf{n}$ & Media & Mediana & Desviación estándar & Mínimo & Máximo \\
\hline $\begin{array}{l}\text { 11. La disposición y el número de terminales de Bloomberg en el } \\
\text { campus San Miguel es adecuada. }\end{array}$ & 113 & 6,20 & 7,00 & 2,690 & 1 & 10 \\
\hline 12. La atención de los terminales de Bloomberg es adecuada. & 112 & 7,56 & 8,00 & 2,134 & 2 & 10 \\
\hline
\end{tabular}

\section{Apéndice 2G. Relacionados al rol del docente en el uso del Bloomberg}

\begin{tabular}{|c|c|c|c|c|c|c|}
\hline Ítems & $\mathbf{n}$ & Media & Mediana & Desviación estándar & Mínimo & Máximo \\
\hline $\begin{array}{l}\text { 13. El docente del curso debería usar más el terminal de Bloom- } \\
\text { berg durante las clases. }\end{array}$ & 113 & 8,09 & 8,00 & 1,806 & 3 & 10 \\
\hline $\begin{array}{l}\text { 14. El docente del curso debería realizar al menos un taller } \\
\text { durante el ciclo sobre el uso de Bloomberg. }\end{array}$ & 113 & 9,27 & 10,00 & 1,722 & 2 & 10 \\
\hline
\end{tabular}

\section{Apéndice 3. Modelos de encuestas aplicadas a los estudiantes de los cursos CON273 y CON279}

Encuesta anónima sobre el uso de Bloomberg en el curso ECF CON 273 / ciclo 201X-X

Por favor, antes de iniciar la encuesta, completa la siguiente información:

$>$ Antes de matricularte en este curso, ya habías usado el Bloomberg (SÍ/NO):

$>$ Si la respuesta fue "SÎ", indica dónde, cuándo y en qué curso:

> Número de reservas que hiciste durante el ciclo para el uso del terminal de Bloomberg:

$>$ Del número de reservas que hiciste durante el ciclo, cuántas veces lo utilizaste solo (a):

$>$ Del número de reservas que hiciste durante el ciclo, cuántas veces lo utilizaste en grupo:

A continuación, coloca una calificación a cada enunciado marcando con una "X" un valor en una escala de 1 al 10, donde 1 significa que estás "completamente en desacuerdo"; y 10, "completamente de acuerdo". 
1. Es importante que los estudiantes de la carrera de Contabilidad conozcan y utilicen el Bloomberg como herramienta de análisis e investigación.
a. 1
b. 2
c. 3
d. 4
e. 5
f. 6
g. 7
h. 8
i. 9
j. 10

2. Es importante que los estudiantes de la carrera de Contabilidad conozcan y utilicen el Bloomberg para la toma de decisiones.
a. 1
b. 2
c. 3
d. 4
e. 5
f. 6
g. 7
h. 8
i. 9
j. 10

3. Una de las principales ventajas de contar con esta herramienta de información en cada una de las sesiones de clase es que se dispone de una base de datos a nivel global de empresas y países, así como datos nacionales. La base de datos es actualizada permanentemente en tiempo real; y sus contenidos están disponibles en archivos digitales, ya sea como base de datos cuantitativos y cualitativos, y/o contenidos de audio y video.
a. 1
b. 2
c. 3
d. 4
e. 5
f. 6
g. 7
h. 8
i. 9
j. 10

4. El uso de Bloomberg en las clases y evaluaciones permite contrastar la teoría con la evidencia empírica con un enfoque tanto doméstico como internacional.
a. 1
b. 2
c. 3
d. 4
e. 5
f. 6
g. 7
h. 8
i. 9
j. 10

5. Se debería elaborar una librería de videos en español sobre el uso de Bloomberg y sus aplicaciones en la carrera de Contabilidad.
a. 1
b. 2
c. 3
d. 4
e. 5
f. 6
g. 7
h. 8
i. 9
j. 10

6. El uso de la información proporcionada por Bloomberg en las sesiones de clase, PAIDEIA, y las evaluaciones de control, formativas individuales y grupales contribuyeron a un mejor aprendizaje de los contenidos del sílabo del curso.
a. 1
b. 2
c. 3
d. 4
e. 5
f. 6
g. 7
h. 8
i. 9
j. 10 
7. Es importante que los estudiantes de la carrera de Contabilidad conozcan el Bloomberg University.
a. 1
b. 2
c. 3
d. 4
e. 5
f. 6
g. 7
h. 8
i. 9
j. 10

8. Es importante que los estudiantes realicen la certificación oficial de Bloomberg ("Bloomberg Certification").
a. 1
b. 2
c. 3
d. 4
e. 5
f. 6
g. 7
h. 8
i. 9
j. 10

9. Creo que, si no hubiéramos usado el Bloomberg durante el ciclo, habríamos aprendido menos.
a. 1
b. 2
c. 3
d. 4
e. 5
f. 6
g. 7
h. 8
i. 9
j. 10

11. La disposición y el número de terminales de Bloomberg en el campus San Miguel es adecuada.
a. 1
b. 2
c. 3
d. 4
e. 5
f. 6
g. 7
h. 8
i. 9
j. 10

12. La atención, el soporte y el servicio de reservas de los terminales de Bloomberg en el campus San Miguel son adecuados.
a. 1
b. 2
c. 3
d. 4
e. 5
f. 6
g. 7
h. 8
i. 9
j. 10

13. El docente del curso debería usar más el terminal de Bloomberg durante las clases.
a. 1
b. 2
c. 3
d. 4
e. 5
f. 6
g. 7
h. 8
i. 9
j. 10 
10. Considero que lo aprendido en este curso con el uso de Bloomberg es fundamental para mi formación profesional.
a. 1
b. 2
c. 3
d. 4
e. 5
f. 6
g. 7
h. 8
i. 9
j. 10

14. El docente del curso debería realizar al menos un taller durante el ciclo sobre el uso de Bloomberg.
a. 1
b. 2
c. 3
d. 4
e. 5
f. 6
g. 7
h. 8
i. 9
j. 10

En este espacio puedes escribir comentarios sobre tu experiencia como usuario de Bloomberg en el proceso de enseńanza y aprendizaje. Por ejemplo, ¿es fácil, normal o difícil aprender a manejar el Bloomberg?

Encuesta anónima sobre el uso de Bloomberg en el curso DDI CON 279 / ciclo 201X-X

Favor antes de iniciar la encuesta completa la siguiente información:

> Antes de matricularte en este curso, ya habías usado el Bloomberg (Sí/NO):

$>$ Si la respuesta fue "SÍ", indicar dónde, cuándo y en qué curso:

$>$ Número de reservas que hiciste durante el ciclo para el uso del terminal de Bloomberg:

Del número de reservas que hiciste durante el ciclo, cuántas veces lo utilizaste solo (a):

Del número de reservas que hiciste durante el ciclo, cuántas veces lo utilizaste en grupo:

A continuación, coloca una calificación a cada enunciado marcando con una " $X$ " un valor en una escala de 1 al 10, donde 1 significa que estás "completamente en desacuerdo"; y 10, "completamente de acuerdo".
En este espacio puedes escribir recomendaciones sobre tu expeencia como usuario de Bloomberg en el proceso de enseñanza y zaje. Por ejemplo, ¿en qué otros cursos también se debería usar Bloomberg? ¿Debe enseńarse solo en los cursos de la carrera Contabilidad o debería empezar a usarse desde EEGGLL? ¿El profesor y/o los jefes de prácticas deben dar más soporte en el uso de Bloomberg? 
1. Es importante que los estudiantes de la carrera de Contabilidad conozcan y utilicen el Bloomberg como herramienta de análisis e investigación.
a. 1
b. 2
c. 3
d. 4
e. 5
f. 6
g. 7
h. 8
i. 9
j. 10

2. Es importante que los estudiantes de la carrera de Contabilidad conozcan y utilicen el Bloomberg para la toma de decisiones.
a. 1
b. 2
c. 3
d. 4
e. 5
f. 6
g. 7
h. 8
i. 9
j. 10

3. Una de las principales ventajas de contar con esta herramienta de información en cada una de las sesiones de clase es que se dispone de una base de datos a nivel global de empresas y países, así como datos nacionales. La base de datos es actualizada permanentemente en tiempo real; y sus contenidos están disponibles en archivos digitales, ya sean como base de datos cuantitativos y cualitativos y/o contenidos de audio y video.
a. 1
b. 2
c. 3
d. 4
e. 5
f. 6
g. 7
h. 8
i. 9
j. 10

4. El uso de Bloomberg en las clases y evaluaciones permite contrastar la teoría con la evidencia empírica con un enfoque tanto doméstico como internacional.
a. 1
b. 2
c. 3
d. 4
e. 5
f. 6
g. 7
h. 8
i. 9
j. 10

5. Se debería elaborar una librería de videos en español sobre el uso de Bloomberg y sus aplicaciones en la carrera de Contabilidad.
a. 1
b. 2
c. 3
d. 4
e. 5
f. 6
g. 7
h. 8
i. 9
j. 10

6. El uso de la información proporcionada por Bloomberg en las sesiones de clase, PAIDEIA, y las evaluaciones de control, formativas individuales y grupales contribuyeron a un mejor aprendizaje de los contenidos del sílabo del curso.
a. 1
b. 2
c. 3
d. 4
e. 5
f. 6
g. 7
h. 8
i. 9
j. 10 
7. Es importante que los estudiantes de la carrera de Contabilidad conozcan el Bloomberg University.
a. 1
b. 2
c. 3
d. 4
e. 5
f. 6
g. 7
h. 8
i. 9
j. 10

8. Es importante que los estudiantes realicen la certificación oficial de Bloomberg ("Bloomberg Certification").
a. 1
b. 2
c. 3
d. 4
e. 5
f. 6
g. 7
h. 8
i. 9
j. 10

8. Creo que, si no hubiéramos usado el Bloomberg durante el ciclo, habría aprendido menos.
a. 1
b. 2
c. 3
d. 4
e. 5
f. 6
g. 7
h. 8
i. 9
j. 10

11. La disposición y el número de terminales de Bloomberg en el campus San Miguel es adecuada.
a. 1
b. 2
c. 3
d. 4
e. 5
f. 6
g. 7
h. 8
i. 9
j. 10

12. La atención, el soporte y el servicio de reservas de los terminales de Bloomberg en el campus San Miguel son adecuados.
a. 1
b. 2
c. 3
d. 4
e. 5
f. 6
g. 7
h. 8
i. 9
j. 10

13. El docente del curso debería usar más el terminal de Bloomberg durante las clases.
a. 1
b. 2
c. 3
d. 4
e. 5
f. 6
g. 7
h. 8
i. 9
j. 10 
9. Considero que lo aprendido en este curso con el uso de Bloomberg es fundamental para mi formación profesional.
a. 1
b. 2
c. 3
d. 4
e. 5
f. 6
g. 7
h. 8
i. 9
j. 10

14. El docente del curso debería realizar al menos un taller durante el ciclo sobre el uso de Bloomberg.
a. 1
b. 2
c. 3
d. 4
e. 5
f. 6
g. 7
h. 8
i. 9
j. 10

En este espacio puedes escribir comentarios sobre tu experiencia como usuario de Bloomberg en el proceso de enseñanza y aprendizaje. Por ejemplo, ¿es fácil, normal o difícil aprender a manejar el Bloomberg?
En este espacio puedes escribir recomendaciones sobre tu experiencia como usuario de Bloomberg en el proceso de enseñanza y aprendizaje. Por ejemplo, ¿en qué otros cursos también se debería usar Bloomberg? ¿Debe enseñarse solo en los cursos de la carrera de Contabilidad o debería empezar a usarse desde EEGGLL? ¿El profesor y/o los jefes de prácticas deben dar más soporte en el uso de Bloomberg? 